\title{
Implications of the $\gamma$-ray Polarization of GRB 021206
}

\author{
Ehud Nakar ${ }^{1}$, Tsvi Piran ${ }^{1}$ and Eli Waxman ${ }^{2,3}$
}

\begin{abstract}
We compare two possible scenarios for the producing of high level of polarization within the prompt emission of a GRB: synchrotron emission from a relativistic jet with a uniform (in space and time) magnetic field and synchrotron emission from a jet with a random magnetic field in the plane of the shock. Somewhat surprisingly we find that both scenarios can produce a comparable level of polarization $(\sim 45-50 \%$ for the uniform field and $\sim 30-35 \%$ for a random field). Uniform time independent field most naturally arises by expansion of the field from the compact object. It requires a $10^{12} \mathrm{G}$ field at the source and a transport of the field as $\propto R^{-1}$. It does not imply Poynting flux domination of the energy of the wind. There is a serious difficulty however, within this scenario, accounting for particle acceleration (which requires random magnetic fields) both for Poynting flux and non-Poynting flux domination. Significant polarization can also arise from a random field provided that the observer is located within $1 / \Gamma$ orientation from a narrow $\left(\theta_{j} \sim 1 / \Gamma\right)$ jet. While most jets are wider, the jet of GRB 021206 from which strong polarization was recently observed, was most likely very narrow. GRB 021206 is among the strongest bursts ever. Adopting the energy-angle relation we find an estimated angle of $<1 / 40 \mathrm{rad}$ or even smaller. Thus, for this particular burst the required geometry is not unusual. We conclude that the RHESSI observations suggest that the prompt emission results from synchrotron radiation. However, in view of the comparable levels of polarizations predicted by both the random field and the homogeneous field scenarios these observations are insufficient to rule out or confirm either one.
\end{abstract}

\section{Introduction}

It is widely accepted that $\gamma$-ray bursts are produced by the dissipation of energy in a highly relativistic wind, driven by gravitational collapse of a (few) solar mass object into a neutron star

\footnotetext{
${ }^{1}$ Racah Institute for Physics, The Hebrew University, Jerusalem 91904, Israel; udini@phys.huji.ac.il, tsvi@phys.huji.ac.il

${ }^{2}$ Department of Condensed Matter Physics, Weizmann Institute, Rehovot 76100, Israel; waxman@wicc.weizmann.ac.il

${ }^{3}$ Incumbent of the Beracha foundation career development chair
} 
or a black hole (see e.g. (Piran 2000; Mészáros 2002) for reviews). As the observed radiation is produced at a large distance from the collapsing object, key questions remain unanswered. The nature of the collapsing object ("inner engine") remains unknown, and the mechanisms responsible for conversion of gravitational energy into a relativistic wind, as well as for gamma-ray production, are not well understood.

The recent detection of very high linear polarization during the prompt $\gamma$-ray emission of GRB 021206 (Coburn \& Boggs 2003; hereafter CB03) has important implications related to these open questions (Waxman 2003; Lyutikov et al. 2003, hereafter LPB03; Granot 2003, hereafter G03,). First, it strongly suggests that synchrotron emission is the basic mechanism operating in GRBs (see however, Eichler and Levinson, 2003, who suggest that a large polarization can arise from Compton scattering of the observed radiation by cold electrons within a very specific geometrical construction.). Second, based on the observed very high degree of polarization $\Pi=(80 \pm 20) \%$, several recent papers claim that a coherent magnetic field is a must (CB03; LPB03), or at least is strongly favored over a random magnetic field (G03). The latter conclusion has also led to claims that the high polarization level measured implies that the magnetic field must be advected from the "inner engine" (LPB03), and that consequently the relativistic flow must be Poynting flux dominated. On the other hand, it was suggested (Waxman 2003) that the high degree of polarization may also arise for a random magnetic field, in case that the GRB outflow is a narrow jet, of opening angle $\theta_{j} \sim 1 / \Gamma$ where $\Gamma$ is the jet Lorentz factor, provided the jet axis is at an angle $\sim 1 / \Gamma$ with respect to the line of sight (Gruzinov 2001).

The main goal of this paper is to critically discuss the implications to GRB models of the observed high degree of polarization. In $\S 2$ we discuss the observations and the constraints they impose on the degree of $\gamma$-ray polarization. In $\S 3$ we present calculations of polarization from jets with a uniform magnetic field (§ 3.1), and with a random magnetic field( $(3.2)$. We present more comprehensive calculation than those of LPB03 and G03, taking into account time dependence, realistic (no-sharp-edge) jet structure, and using the specific electrons' distribution which is relevant to the observation of RHESSI. We find that the observed polarization may be explained assuming either a uniform or a random magnetic field configuration. The constraints imposed on the model in each case are, however, different. We discuss the implications under the hypothesis of a uniform field, in particular the issue of whether a uniform field necessarily favors magnetically dominated out flow, in $\S 4$. Implications under the hypothesis of a random field, in particular the issue of whether the required $\sim 1 / \Gamma$ offset between the jet axis and the line-of-sight is likely or not, are discussed in $\S 5$. Our conclusions are summarized in $\S 6$.

\section{Discussion of the observations}

The data analysis of CB03 is based on 12 data points which are collected over 5sec. Each of these points is a sum of several independent observations taken at different times. Thus the data

is some kind of convolution of the polarization over the whole duration of the burst and should be 
considered as a time averaged data.

CB03 test two hypothesis with this data. First they test the null hypothesis of no polarization. This hypothesis is rejected at a confidence level of $5.7 \sigma$. It is clear that the emission is strongly polarized. Second they estimate the modulation factor assuming a constant polarization direction and angle during the whole burst. The best fit to the data is achieved with $\Pi=(80 \pm 20) \%$. However, CB03 find that the probability that $\chi^{2}$ is greater than the value obtained with this fit is $5 \%$.

In checking the second hypothesis, CB03 assumed a time independent polarization as well as a time independent photon spectrum (given by the photon spectrum averaged over the GRB duration). The detected polarization signal arises from a correlation between the time dependence of scattered photon flux and the angular orientation of the satellite, which varies on a time scale similar to the burst duration (the satellite rotation period is comparable to burst duration). Since both the photon spectrum and the polarization are likely to vary over this time scale, it is not clear what the lower limit implied by the observation on the polarization level is. A robust estimate of this limit will require a comparison of the observed signal with that obtained under various assumptions regarding the time dependence of the spectrum and of the polarization.

Our interpretation of the available analysis of the observations is that the time integrated polarization of the prompt $\gamma$-ray emission is of the order of tens of percents, and that the polarization angle does not vary significantly during the whole burst.

\section{Synchrotron Polarization from Relativistic Moving Jets}

The level of polarization produced by an ordered magnetic field that is in the plane of the shock was calculated by LPB03 and Granot \& Konigel (2003), while G03 calculates the polarization both from random and from ordered magnetic fields (see also Sari (1999) and Ghisellini \& Lazzati, (1999)) . Below we repeat these calculations with some minor changes that are relevant for the interpretation of RHESSI's observation. We also add a time dependent calculation that shows the variation of the polarization with time. The energy range in which the polarization is measured is 0.15 - $2 \mathrm{Mev}$. We expect that this arises from a fast cooling synchrotron emission and it is above both the synchrotron and the cooling frequencies. The spectral index $\left(F_{\nu} \propto \nu^{-\alpha}\right)$ in this power law

segment is $\alpha \approx 1$, while the dependence of the intensity on the magnetic field in the fluid comoving frame is $I_{\nu^{\prime}}^{\prime} \propto B^{\varepsilon}$, with $\varepsilon=(p-2) / 2 \approx 0$ and $p \approx 2-2.5$ the power index of the electron's energy distribution. The local emitted polarization of a synchrotron emission at this power law segment is $\Pi_{\text {synch }}=(p+2) /(p+10 / 3) \approx 75 \%$ (G03). Averaging over the emitting area reduces the polarization and in the following discussion we will estimate the resulting polarization due to this averaging relative to $\Pi_{\text {synch }}$. Namely, unless otherwise specified the results should be multiplied by this factor.

We consider two cases: ordered (homogeneous) magnetic field and a random magnetic field. In 
both cases the magnetic fields are in the plane of the shock.

\subsection{Homogeneous Magnetic Field}

Consider a top-hat jet with an opening angle $\theta_{j}$ propagating at Lorentz factor $\Gamma$ observed from $\theta_{o b s} \ll \theta_{j}{ }^{4}$ with a constant magnetic field in the plane perpendicular to the motion. The observed stokes parameters are weighted averages of the local stokes parameters at different regions of the shell. The instantaneous polarization is calculated using the instantaneous observed flux $F_{\nu}(y, T) \propto(1+y)^{-(3+\alpha)}$ as the weights, where $y \equiv(\Gamma \theta)^{2}$ and $T$ is the observer time. The time integrated polarization is calculated using the fluences as weights: $\int_{0}^{\infty} F_{\nu}(y, T) d T \propto(1+y)^{-(2+\alpha)}$.

For $\varepsilon=0$ the time integrated stokes parameters (note that $V=0$ as the polarization is linear) and polarization are given by:

$$
\frac{\left\{\begin{array}{l}
Q \\
U
\end{array}\right\}}{I}=\Pi_{\text {synch }} \frac{\int_{0}^{2 \pi} \int_{0}^{\infty}(1+y)^{-(2+\alpha)}\left\{\begin{array}{c}
\cos \left(2 \theta_{p}\right) \\
\sin \left(2 \theta_{p}\right)
\end{array}\right\} d y d \phi}{\int_{0}^{2 \pi} \int_{0}^{\infty}(1+y)^{-(2+\alpha)} d y d \phi}
$$

and

$$
\Pi=\frac{\sqrt{U^{2}+Q^{2}}}{I}
$$

where $\theta_{p}=\phi+\arctan \left(\frac{1-y}{1+y} \cot \phi\right)$ (Granot \& Konigel 2003). This result is similar to the result of LPB03 apart for the fact that LPB03 calculate the polarization for a slow cooling emission with $\nu_{m}<\nu<\nu_{c}$, (with different values of $\alpha$ and $\epsilon$ ) which is not the relevant case for the prompt $\gamma$-ray emission that was observed by RHESSI. For $\alpha=1$ Eqs. 1-2 yield a polarization level of $\Pi / \Pi_{\text {synch }} \approx 60 \%$. I.e. $60 \%$ of the maximal synchrotron polarization, or an overall polarization of $\sim 45 \%$ (taking the exact values of $\alpha$ and $\epsilon$ for $p=2.5$ results in an overall polarization of $\sim 50 \%$ ).

The time dependent polarization depends on the the temporal properties of the emitting area. We consider the simplest case of a thin shell radiating at a constant luminosity between $R_{1}$ and $R_{2}$. The first photon arrives to the observer at $T_{1}=R_{1} /\left(2 c \Gamma^{2}\right)$. At this time the observer sees only photons from the center $(y=0)$, thus the initial polarization is $100 \%$. Later the observer receives photons both from the center and from higher angles and the polarization drops to an asymptotic value which is reached when photons from the whole shell reach the observer simultaneously. Finally, during the decay of the observed pulse the observer receives only photons from $y>0$ and the polarization level drops. More precisely at time $T$ the observer receive photons from $y_{\min }<y<$ $y_{\max }$ where:

$$
\begin{gathered}
y_{\min }(\widetilde{T})=\max \left(0, \widetilde{T} / \widetilde{T_{2}}-1\right) \\
y_{\max }(\widetilde{T})=\widetilde{T}-1
\end{gathered},
$$

\footnotetext{
${ }^{4}$ Through the paper we work in spherical co-ordinates with the origin at the source of the explosion. $\theta$ is the angle with respect to the line between the observer and the origin, and $\phi$ is the azimuthal angel.
} 
where $\widetilde{T}=T / T_{1}$ and $\widetilde{T_{2}}=R_{2} / R_{1}$. Now the polarization at $\widetilde{T}$ is given by:

$$
\frac{\left\{\begin{array}{l}
Q \\
U
\end{array}\right\}}{I}(\widetilde{T})=\frac{\int_{0}^{2 \pi} \int_{y_{\min }}^{y_{\max }}(1+y)^{-(3+\alpha)}\left\{\begin{array}{c}
\cos \left(2 \theta_{p}\right) \\
\sin \left(2 \theta_{p}\right)
\end{array}\right\} d y d \phi}{\int_{0}^{2 \pi} \int_{y_{\min }}^{y_{\max }}(1+y)^{-(3+\alpha)} d y d \phi} \Pi_{\text {synch }} .
$$

Figure 1 depicts the time dependent polarization from a shell emitting between $R_{1}$ and $R_{2}$. When $\left(R_{2}-R_{1}\right) / R_{1} \ll 1$ (the radial time, $\left(R_{2}-R_{1}\right) / 2 c \Gamma^{2}$, is much shorter than the angular time $\left(R_{1} /\left(2 c \Gamma^{2}\right)\right)$, left panel, the polarization level drops from $100 \%$ to a few percent within one angular time. When the radial time is much larger than the angular time, right panel, the polarization level drops from $100 \%$ to an asymptotic value until the peak of the pulse. As the pulse begins to decay the polarization drops to a few percent within one angular time of $R_{2}$.

\subsection{Polarization from a Random Magnetic Field}

Waxman (2003) has suggested that a high polarization level can be obtained when a narrow jet that is observed from the edge even if the magnetic field is random. We consider here a random magnetic field that remains planner in the plane of the shock. For a three dimensional random magnetic field the polarization essentially vanishes. For $\epsilon=(p-2) / 2 \approx 0$ the degree of observed polarization of the emission emitted from a small region at angle $y$ is: $\Pi(y) / \Pi_{\text {synch }}=\min (y, 1 / y)$. The overall time integrated stokes parameters are:

$$
\frac{\left\{\begin{array}{l}
Q \\
U
\end{array}\right\}}{I}=\Pi_{\text {synch }} \frac{\int_{0}^{2 \pi} \int_{0}^{\infty} P_{\nu^{\prime}, m}^{\prime}(1+y)^{-(2+\alpha)} \min (y, 1 / y)\left\{\begin{array}{c}
\cos (2 \phi) \\
\sin (2 \phi)
\end{array}\right\} d y d \phi}{\int_{0}^{2 \pi} \int_{0}^{\infty} P_{\nu^{\prime}, m}^{\prime}(1+y)^{-(2+\alpha)} d y d \phi},
$$

where $P_{\nu^{\prime}, m}^{\prime}=P_{\nu^{\prime}, m}^{\prime}(y, \phi)$ is the emitted power at the synchrotron frequency in the fluid rest frame. For a top-hat jet with sharp edges $P_{\nu^{\prime}, m}^{\prime}$ is constant for any $y$ and $\phi$ within the jet and zero otherwise. For a structured jet $P_{\nu^{\prime}, m}^{\prime}$ depends on the angle from the jet axis.

The maximal polarization is observed when we see the edge of the jet. The probability to see the edge of a top-hat jet with sharp edges and an opening angle $\theta_{j} \Gamma \gg 1$ is negligible. On the other hand a jet with $\theta_{j} \Gamma \ll 1$ is not expected. Thus the only physical cases in which we can expect a large polarization are $1 \lesssim \theta_{j} \Gamma<$ a few .

Figure 2 depicts the time integrated polarization and the efficiency from sharp edged jets with different opening angles as a function of the the angle between the jet axis and the line of sight, $\theta_{\text {obs }}$. The efficiency, $e_{f f}$ is defined to be the ratio between the observed fluence at $\theta_{\text {obs }}$ and the maximal possible observed fluence at $\theta_{o b s}=0$. In all these cases the polarization is peaked above $40 \%$, however the efficiency decrease sharply as the polarization increase. Thus the probability to see high polarization grows when $\theta_{j}$ decrease. The probability that $\theta_{o b s}$ is such that the polarization is 

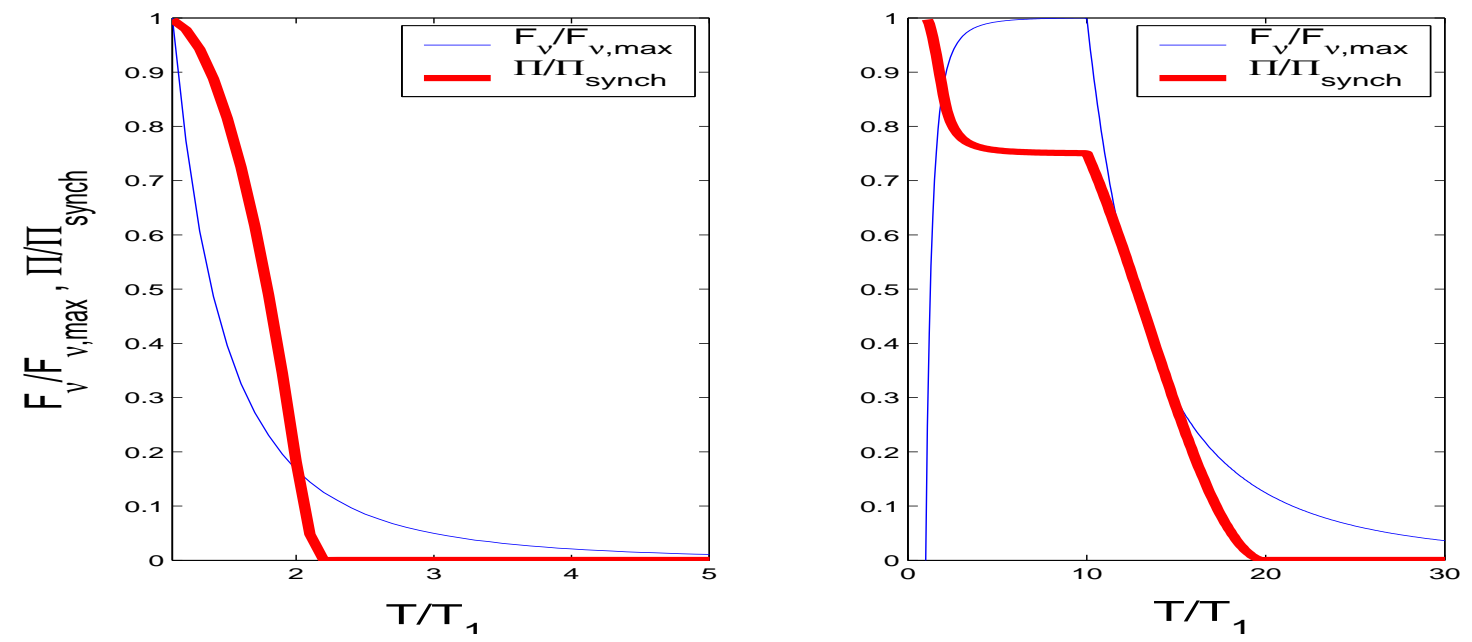

Fig. 1.- The instantaneous polarization (thick line) and normalized flux (thin line) from a thin shell propagating at a Lorentz factor $\Gamma$ and radiating constantly between the radii $R_{1}$ and $R_{2}$ under the hypothesis of uniform and constant magnetic field. The $\mathrm{x}$ axis is the observer time $T$ in units of the angular time at $R_{1}: T_{1}=R_{1} /\left(2 c \Gamma^{2}\right)$. In the left panel $R_{2}=1.01 R_{1}$, in the right panel $R_{2}=10 R_{1}$ and we can see the gradual increase in flux and the corresponding decrease in the polarization that reaches an asymptotic value when the whole shell is observed.
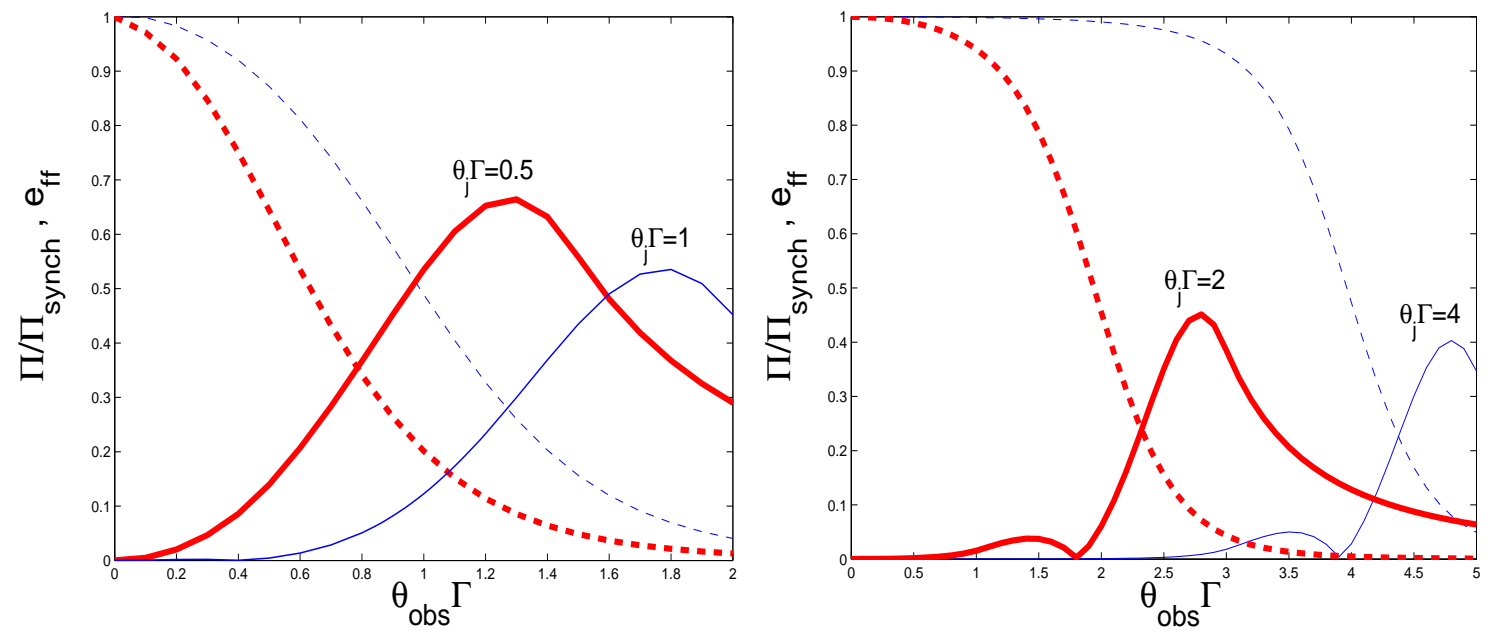

Fig. 2.- The time integrated polarization (solid line) and the efficiency (dashed line) as a function of $\theta_{o b s} \Gamma$ for four different values of $\theta_{j}$ for a random magnetic field. 
larger than $30 \%\left(\cdot \Pi_{\text {synch }}\right)$ while $e_{f f}>0.1$ is $0.68,0.41,0.2 \& 0.08$ for $\theta_{j} \Gamma=0.5,1,2,4$ respectively. In reality this probability will be smaller, as the chance to observe a burst increases with its observed flux.

Figure 3a depict the polarization produced by a jet with exponential wings. The emitted power of the jet as a function of the angle with respect to the jet axis, $\psi$ is:

$$
P_{\nu^{\prime}, m}^{\prime}=\left\{\begin{array}{c}
P_{\nu^{\prime}, m}^{\prime}(\psi=0) \quad \psi \Gamma<1 \\
P_{\nu^{\prime}, m}^{\prime}(\psi=0) \exp [(\psi \Gamma-1) / \Delta] \quad \psi \Gamma>1
\end{array},\right.
$$

where $\Delta$ is the characteristic angle (in units of $1 / \Gamma$ ) over which the emission decays. According to Fig.3a it is clear that in order to obtain high polarization the wings of the jet must be sharp $(\Delta<0.25)$. An alternative possible wing is a power law wing:

$$
P_{\nu^{\prime}, m}^{\prime}=\left\{\begin{array}{c}
P_{\nu^{\prime}, m}^{\prime}(\psi=0) \quad \psi<\psi_{c} \\
P_{\nu^{\prime}, m}^{\prime}(\psi=0)\left(\psi / \psi_{c}\right)^{-\delta} \quad \psi>\psi_{c}
\end{array}\right.
$$

The case of $\delta=2$ is especially interesting as it is the jet structure of the universal standard jet $(\text { Rossi et al. 2002) })^{5}$. Due to similar considerations as above $\psi_{c} \Gamma \gtrsim 1$. Figure 3 b shows that $\psi_{c} \Gamma=1$ can not produce a large polarization.

Similarly to the ordered magnetic field case, the time dependent polarization depends on the the temporal properties of the emitting area. Again we consider the simplest case of a thin shell which radiates at constant luminosity between $R_{1}$ and $R_{2}$. The calculation is also similar to the case of ordered magnetic field:

$$
\frac{\left\{\begin{array}{c}
Q \\
U
\end{array}\right\}}{I}(\widetilde{T})=\Pi_{\text {synch }} \frac{\int_{0}^{2 \pi} \int_{y_{\min }}^{y_{\max }} P_{\nu^{\prime}, m}^{\prime}(1+y)^{-(3+\alpha)} \min (y, 1 / y)\left\{\begin{array}{c}
\cos (2 \phi) \\
\sin (2 \phi)
\end{array}\right\} d y d \phi}{\int_{0}^{2 \pi} \int_{y_{\min }}^{y_{\max }} P_{\nu^{\prime}, m}^{\prime}(1+y)^{-(3+\alpha)} d y d \phi},
$$

where $y_{\min , \max }$ are defined in Eq. 3. Figure 4 depicts the time dependent polarization for a top-hat jet with $\theta_{j} \Gamma=1$, observed from $\theta_{o b s} \Gamma=1.8$. When the radial time is much shorter than the angular time, left panel, the polarization level follows the flux of the pulse and at its peak it can reach more then $80 \%\left(\cdot \Pi_{\text {synch }}\right)$. When the radial time is much larger than the angular time, right panel, the polarization level rises at first and than it drops to an asymptotic level till the peak of the pulse. As the pulse starts to decay, the polarization resumes its decay as well.

So far we have assumed that the Lorentz factor, $\Gamma$ does not vary during the burst. Large variation in the Lorentz factor will induce variation in the averaging regions and will reduce the

\footnotetext{
${ }^{5}$ Note that in the universal standard jet this is the profile of the energy and not of the emitted power. However if the radiated efficiency is constant both profiles are similar.
} 

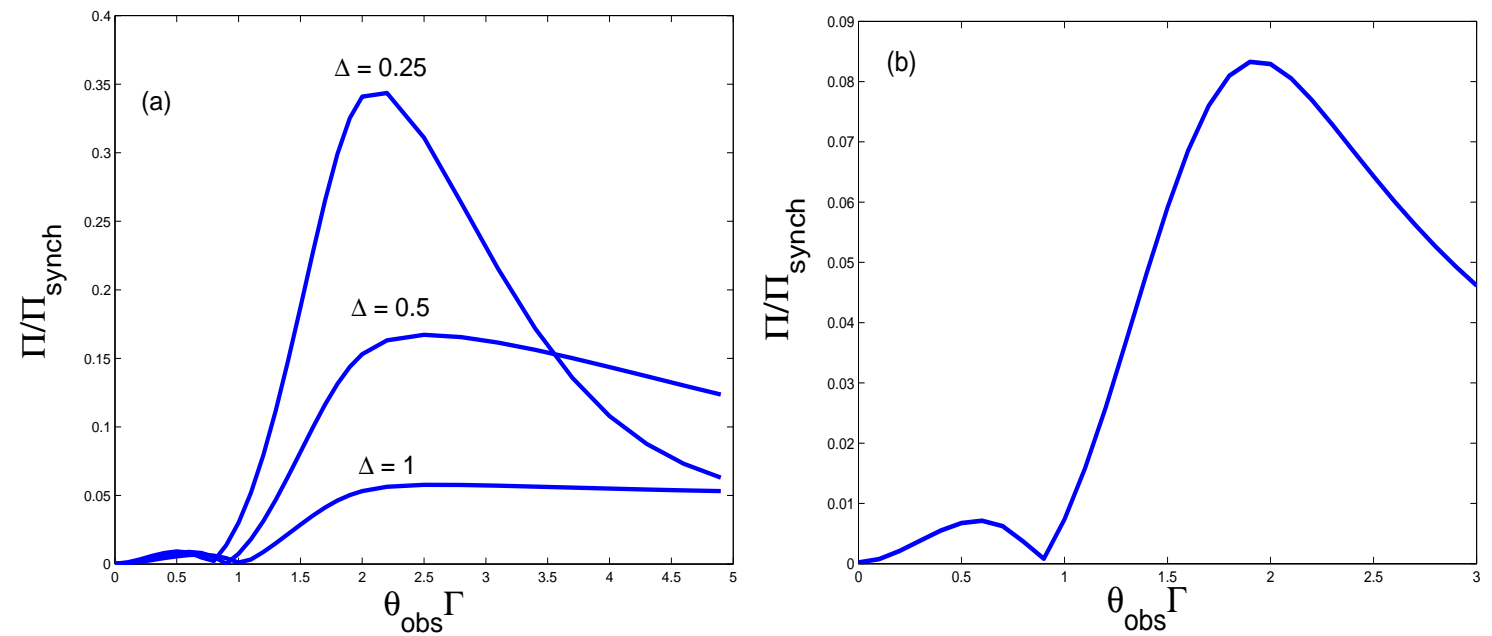

Fig. 3.- The time integrated polarization of a jet with exponantial wings (left panel, according to Eq. 6) and power-law wings (right panel, according to Eq. 7), as a function of $\theta_{\text {obs }}$. The size of the core of the jet (over which the emissivity is constant) is $1 / \Gamma$.
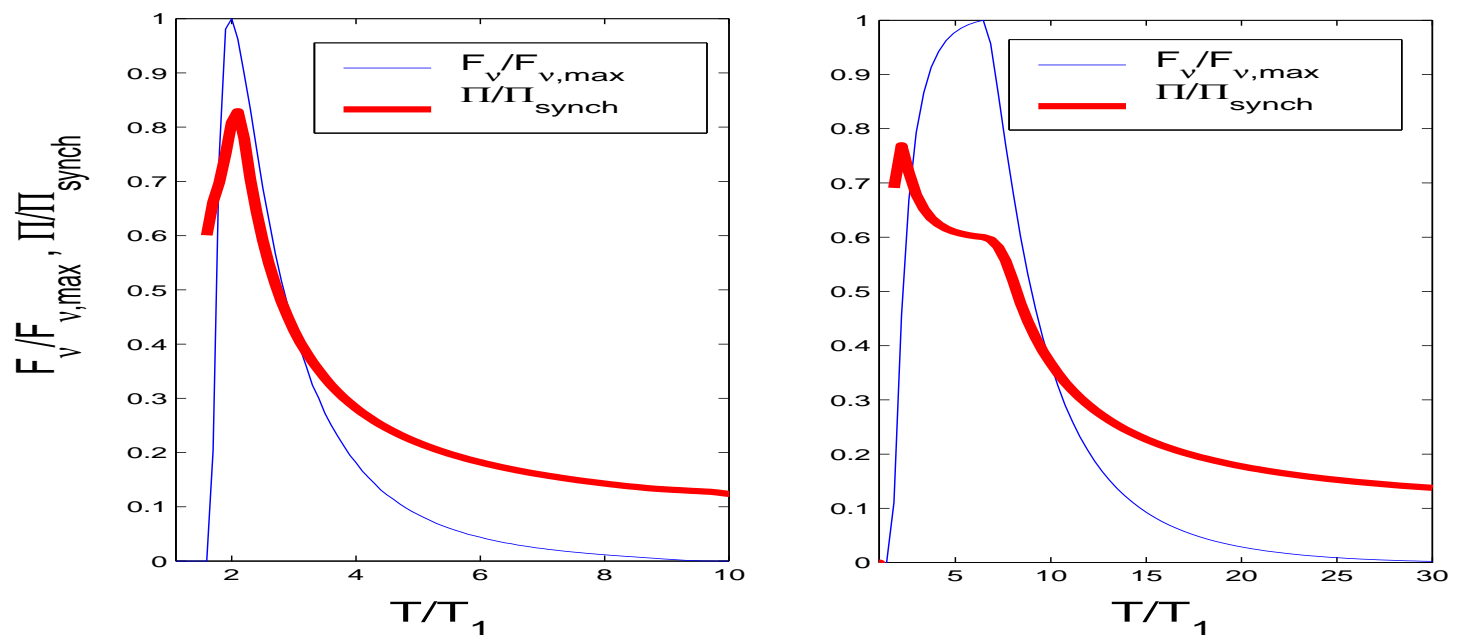

Fig. 4.- The instantaneous polarization (thick line) and normalized flux (thin line) from a thin shell which propagates at a Lorentz factor $\Gamma$ and radiates constantly between the radii $R_{1}$ and $R_{2}$ under the hypothesis of random magnetic field in the plane of the shock. The $\mathrm{x}$ axis is the observer time $T$ in units of the angular time at $R_{1}: T_{1}=R_{1} /\left(2 c \Gamma^{2}\right)$. In the left panel $R_{2}=1.1 R_{1}$, in the right panel $R_{2}=4 R_{1}$. 
observed polarization by a factor of a few (G03), making it too low. However, if the modulated wind is a constant energy flow (which is favored due to other reasons (Nakar \& Piran 2002)) then the spread of the Lorentz factor of the emitting regions is small, and no significant reduction in the level of polarization is expected. Furthermore, even if there are variations in the Lorentz factor we would expect that the variations in the flux weighted Loretnz factor will be rather small.

\section{Implications for a uniform magnetic field}

We discuss in this section the implications of the high level of polarization, under the hypothesis of a uniform magnetic field configuration. We first show ( $§ 4.1)$ that "uniformity" means "uniform field over a causally connected region" of the outflow, and then discuss the implications $(\S 4.2, \S 4.3)$.

\subsection{Coherence scale and causal connection}

A distant observer receives radiation from a conical section of the outflow, of opening angle $\sim 1 / \Gamma$ where $\Gamma$ is the Lorentz factor of the out flowing plasma. The transverse size of the observed region is therefore $\sim R / \Gamma$, where $R$ is the radius at which radiation is produced. This length scale is comparable to the size of a causally connected region within the flow, as demonstrated by the following simple argument.

Consider a signal emitted at radius $R_{0}$ and propagating at a speed $\beta_{s} c$, where $c$ is the speed of light, in the plasma rest frame. By the time the plasma expands to radius $2 R_{0}$, the time that elapsed in the plasma frame is $R_{0} / \Gamma c$, and hence the distance travelled by the signal is $\beta_{s} R_{0} / \Gamma$, i.e. the transversal size over which the signal has been "communicated" is $\beta_{s} R_{0} / \Gamma$. Since this transverse size is linear in $R_{0}$ for doubling of $R$, it follows that distance travelled by the signal grows logarithmically with $R$, and is of order $\beta_{s}[R / \Gamma] \log \left(R / R_{0}\right)$. This result is independent of the nature of the flow, and holds regardless of whether the energy density in the outflow is carried mainly by magnetic field or particles. It is simply a results of causality. Note that LPB03 base their criticism of the hydrodynamic scenario on a different calculation of the size of the causally connected regions. They consider signals that propagate at a constant speed in the observer frame (and ignore the fact that a hydorodynamic wave is dragged along the flow) and obtain a different result. Our result implies that under the "uniform field" scenario, the field is required to be uniform over causally connected regions both in hydrodynamic flows and in Poynting flux dominated flows.

\subsection{Transport from the source}

There are two qualitatively different scenarios for the production of the strong magnetic field implied to exist in the synchrotron emitting region. The field may be produced near the compact 
object driving the wind (as originally suggested by (Usov 94)), and then carried by the wind to large radii where radiation is emitted. Alternatively, it may be produced (or strongly amplified) in the radiation emitting region itself. The common mechanism assumed to be responsible for the latter scenario is magnetic field amplification by plasma instabilities in collisionless shocks. The magnetic field produced by the latter process is naturally created on small (skin-depth) scales (e.g. (Gruzinov \& Waxman 1999; Medvedev \& Loeb 1999)), and there is no well understood process that increases the field coherence scale to a size comparable to the that of a causally connected region within the outflow. Nevertheless, afterglow observations strongly suggest that the field is created by the collisionless shocks, and that its coherence length does grow to the size of causally connected regions (Gruzinov \& Waxman 1999). However, if the field is produced by this process, we would expect its direction to vary with time on a time scale similar to that of the time scale for $\gamma$-ray flux variations. In this case the resulting time integrated polarization would be similar to the one produced by a random field for a variable burst as GRB 021206. In order to obtain the large time averaged polarization observed by CB03, under the hypothesis of uniform field, the field must be coherent over causally connected regions and time independent.

The most natural assumption that would lead to a coherent time independent field is that the field is produced by the compact object, and then carried by the wind to large distances. LPB have shown that in the case where the wind is cylindrically symmetric and dominated by Poynting flux, assuming that the inertia of the plasma is negligible ("force free" approximation), the field is dominated at large distances by a coherent toroidal component, $B_{\phi}$. In this case, large polarization will arise if the expanding plasma is observed at an angle $>1 / \Gamma$ with respect to the symmetry axis. Note that this torodial geometry will keep the direction of the magnetic field constant in time, as required under the uniform field hypothesis, even if the wind is not steady.

However, coherent toroidal field may also be obtained in the case where the energy density is not dominated by Poynting flux. In the case where the wind energy density is dominated by the plasma particles, and where the conductivity is high so that the electric field in the plasma rest frame $\left(\vec{E}^{\prime}=\vec{E}+c^{-1} \vec{v} \times \vec{B}\right)$ is vanishingly small (the MHD approximation), Maxwell's eqs. give, for a steady wind, $\nabla \times(\vec{v} \times \vec{B})=0$, which imply $B_{\phi} \propto R^{-1}$ for uniform radial velocity. Thus, in this case too a coherent toroidal field dominates at large radii, with energy flux that is radius independent. In order to account for the observed $\gamma$-ray emission it is sufficient that the magnetic field carries $<1 \%$ of the wind luminosity.

\subsection{Particle acceleration}

Regardless of whether or not the wind energy density is dominated by Poynting flux, the observed $\gamma$-rays are produced by the dissipation of wind energy, which converts either magnetic field energy or plasma kinetic energy to high energy particles (electrons), which then radiate away the dissipated energy. In the kinetic energy dissipation scenario, particles are assumed to be accelerated in collisionless shocks. In this case, non uniform field components must be produced in the shocks, 
since particles of a wide range of energies and Larmor radii must be efficiently scattered across the shock. It may therefore appear that particle acceleration and uniform magnetic field are mutually exclusive.

A similar problem arises also in the case of a Poynting flux dominated flow. LPB do not present a model for the dissipation of magnetic field energy and particle acceleration. However, it appears to be unlikely that such dissipation, which converts a significant fraction of field energy to particle energy, may arise without exciting waves of a wide range of wavelengths and wave vector orientations resulting in a large fraction of the energy density converted to a random field component. (Smolsky \& Usov 2000) propose a specific mechanism for magnetic field energy dissipation. However, since their calculations are one dimensional, it is difficult to draw conclusions regarding the resulting polarization.

A possible solution to this apparent contradiction between uniform field and particle acceleration is that a non negligible fraction of the magnetic field remains coherent (and responsible for the polarization) while a random component with non negligible fraction of the energy density is produced via dissipation and is responsible for particle acceleration. However, in this configuration the radiation from the random component of the magnetic field would reduce the polarization level. A second solution may be a separation of the acceleration region and the emission regions. However, no detailed physical solution of this type has been presented so far.

\section{Implications for a random magnetic field}

An off-axis observation can produce, in the case of a random magnetic field configuration, up to $60 \%\left(\cdot \Pi_{\text {synch }}\right)$ time integrated polarization (although a value of $30 \%\left(\cdot \Pi_{\text {synch }}\right)$ is much more probable) and an instantaneous polarization which can peak around $80 \%$ (see $\S 3.2$ ). These results are quite similar to the polarization level that is produced by a uniform time independent magnetic field. While the main difficulty in the uniform time independent field scenario is the production of the field ( $(4.2)$ and the particle acceleration ( $(4.3)$ within such a field, the physical conditions which may produce a random magnetic field are much more relaxed. However, the main drawback of the random magnetic field scenario is that not all the observers see the same level of polarization. In other words, a high degree of polarization during the prompt emission is possible only if $\theta_{\text {obs }} \approx$ $\theta_{j} \approx 1 / \Gamma \approx 0.01 \mathrm{rad}$. Such orientation may appear unlikely. However, we argue below that this is not necessarily the case in GRB 021206.

The opening angle of GRB 021206 can be estimated using the constant total energy of GRBs (Frail et al. 2001; Panaitescu \& Kumar 2001). The observed fluence of GRB 021206 was 1.6 . $10^{-4} \mathrm{ergs} / \mathrm{cm}^{2}$ at the energy range of $25-100 \mathrm{Kev}$ (Hurley et al. 2002). This puts GRB 021206 as one of the most powerfull bursts, and the most powerful one (a factor of 2-3 above GRB990123) after correcting for the fact that it was observed only in a narrow band (compared to the wide BATSE band of 20-2000keV). We correct for this factor using the data for GRB 020813 that was 
also observed by the IPN at the same waveband. This burst that was at $\mathrm{z}=1.25$ had an average total (beaming corrected) energy of $1.2 \cdot 10^{51} \mathrm{ergs}$ (which is the typical energy of GRBs), a fluence lower by a factor of four (compared to GRB 021206) and had an opening angle of $3^{\circ}$ (see Tables 1 and 2 of Bloom et al. (2003)). Assuming, conservatively, that the redshift of GRB 021206 is comparable we find $\theta_{j} \approx 1.5^{\circ} \approx 1 / 40 \mathrm{rad}$, putting GRB 021206 as the narrowest jet seen so far (a factor of 2 below the narrowest jets found by Bloom et al. (2003)). Note that the correction for varying the redshift in the range 1-2 is small.

This simple estimate of the opening angle yields $\theta_{j}$ which is a factor 2-3 from the typical (inverse) Lorentz factors seen in GRBs. We can refine the estimate. As we have shown above $(\S 3.2)$ in order to obtain high polarization the jet must be seen from the edge. This implies that we detected only a fraction $e_{f f}$ of the full flux of the burst. With $e_{f f} \approx 0.1$ this will introduce an additional correction of $\sim 1 / 3$ to the opening angle, putting the overall angle around $1 / 120 \mathrm{rad}$. Thus, we find that the condition $\theta_{j} \approx 1 / \Gamma$ is very reasonable for this particular burst. We also find that as GRB 021206 is clearly atypical, as seen from its remarkably high fluence, one does not have to worry too much about the issue of whether the observation of the jet from the edge is generic or not.

\section{Conclusions}

The recent detection of very high linear polarization during the prompt $\gamma$-ray emission of GRB 021206 (CB03) suggests strongly that the $\gamma$-rays are produced by synchrotron emission of relativistic particles in strong magnetic field. The intrinsic linear polarization of such emission can reach $75 \%$ (depending weakly on the details of the electron's energy distribution) and it is perpendicular to the magnetic field and to the photon's momentum. In GRBs the emission arises from a relativistic jet and the observed polarization should be averaged over the emitting region taking into account relativistic effects that rotate the propagation vector and limit the observing angle to a region of a size $\Gamma^{-1}$. This averaging essentially reduces the polarization from its local maximal value. The relativistic rotation of the polarization vector leads to a decrease in the overall polarization even in cases that the whole emitting region is emitting locally fully polarized radiation.

We have calculated the polarization level of synchrotron radiation from relativistic jets with either constant (uniform in space and time independent) or random (but in the plane perpendicular to the motion) magnetic fields. For a homogeneous field one can obtain a maximal time integrated polarization of $60-65 \%$ of the maximal synchrotron polarization i.e. an overall polarization of 45 $50 \%$. It is interesting to note that even for a constant magnetic field the instantaneous polarization level (but not the direction) varies with time and the extend of the temporal variability depend on the ratio of the intrinsic pulse duration to the angular spreading time $R / \Gamma^{2}$ across the jet.

The natural way to produce a time independent homogeneous magnetic field across the jet is to transport the magnetic field from the source (see $\S 4.2$ ). The magnetic field required to produce 
the observed synchrotron emission is rather strong and it implies both a $\sim 10^{12} \mathrm{G}$ magnetic fields at the source and a transport of the field $\propto R^{-1}$. As only the torodial component of the field can be transported as $R^{-1}$ we must be looking at the system at least $1 / \Gamma$ away from the symmetry axis. The magnitudes of the field involved are large but they do not necessarily imply that most of the wind energy has to be carried as a Poynting flux. Moreover, the constrain of a homogeneous magnetic field over the observable region $(1 / \Gamma)$ does not rule out a baryons dominated flow. Thus even if the observed polarization results from a homogeneous magnetic field, it is not enough to distinguish between a Poynting flux and non-Poynting flux dominated flows. There is a serious difficulty, within the homogeneous field model for accounting for particle acceleration both for Poynting flux and non-Poynting flux domination as the standard Fermi acceleration processes require a diffusion in a random magnetic field (see §4.3). Even within an alternative acceleration model based on field reconnection rather than on Fermi process this can be resolved only by separating the acceleration and emission regions, or by producing a co-existing random and ordered magnetic fields.

The maximal time integrated polarization from a random magnetic field depends critically on the viewing angle, $\theta_{o b s}$. It increases with $\theta_{o b s}$, reaches a maximum around $\theta_{o b s}=1 / \Gamma+\theta_{j}$ and decreases to zero. However as the polarization increases when the observer moves outwards, the observer views a smaller fraction of the jet and $e_{f f}$ decreases. With reasonable parameters one can obtain a polarization of $40 \%$ of the maximal synchrotron polarization i.e. an overall polarization of $30 \%$ and $e_{f f} \approx 0.1$. Thus, somewhat surprisingly, a random magnetic field produces almost as strong polarization as the homogeneous one. This arises because in the homogeneous case the center contributes of the higher polarization while the peripheral regions at angle of $1 / \Gamma$ reduce it, while in the random case the peripheral regions contribute to the high polarization while the center reduces it.

The main challenge for the random field scenario is the jet opening angle and orientation (see §5). However, for this particular burst we find that the required geometry is likely if we adopt the energy-angle relation (Frail et al. 2001). The probability to observe the edge of the jet depends on $\theta_{j} \Gamma$. It will be significant only if $\theta_{j} \Gamma \approx 1$ or at least it is not significantly larger than unity. This last point may indicate that the situation of observing large polarization from a jet with random magnetic fields is not generic. However, we have shown that GRB 021206 is not generic. Its fluence put it as one of the strongest GRBs observed ever and according to the constant energy reservoir (Frail et al. 2001) interpretation this implies that $\theta_{j}$ was one of the narrowest. Conservative estimates that put it at $z \approx 1.25$ suggest $\theta_{j} \approx 1 / 40 \mathrm{rad}$ and inclusion of the efficiency factor yield $\theta_{j} \approx 1 / 120 \mathrm{rad}$, values that almost comparable to various estimates of $1 / \Gamma$. We have also found that the jet needs to be reasonably sharp, but not unrealistically so.

Over all we find that both cases are incompatible with the canonical value of $80 \%$ estimated by CB03. However, the data has been fitted with only two models: (i) no polarization that was rejected and (ii) a constant polarization of $80 \%$, which also does not provide a perfect fit to the data. On the other hand both the homogeneous field and the random field scenarios suggest that the polarization degree (and possibly the orientation) vary with time. We conclude that the current 
data indicates that the polarization level was high, suggesting synchrotron that has intrinsic high polarization as the origin of the prompt emission. However, in view of the comparable levels of

polarizations predicted by both the random field and the homogeneous field scenarios the current data is insufficient to rule out or confirm either one.

We thank J. Granot, D. Guetta and A. Lazar and for helpful discussions and comments.

\section{REFERENCES}

Bloom, J. S., Frail D. A., \& Kulkarni, S. R., 2003, Accepted to ApJ, (astro-ph/0302210)

Coburn, W., \& Boggs, S. E., 2003, Nature 423, 415 (CB03)

Eichler, D., \& Levinson, A., 2003, astro-ph/0306360

Frail, D. A., et al., 2001, ApJL, 562 L55

Ghisellini, G. \& Lazzati, D., 1999, MNRAS., 309 L7

Granot, J., 2003, submitted to ApJL(astro-ph/0306322) (G03)

Granot, J., \& Konigel, A., 2003, submitted to ApJL(astro-ph/0304286)

A. Gruzinov, E. Waxman 1999, ApJ 511852

A. Gruzinov 2001, ApJ 56315

Hurley, K., et al., 2002, GCN Circ. 1727, 1728

Lyutikov, M., Pariev, V. I., \& Blandford, R. D., 2003, submitted to ApJ(astro-ph/0305410) (LPB03)

Medvedev, M. V. \& Loeb, A. 1999, Astrophys. J. 526, 697-706

Mészáros, P. 2002, ARA\&A 40, 137

Nakar, E., \& Piran, T., 2002 ApJL, 572, L139

Panaitescu, A. \& Kumar, P., 2001, ApJ, 554667

Piran, T. 2000, Phys. Rep. 333, 529.

Rossi, E., Lazzati, D., \& Rees, M. J., 2002, MNRAS, 332, 945

Sari, R., 1999, ApJ 542 L43

Smolsky, M. V. \& Usov, V. V. 2000, ApJ 531, 764

V.V. Usov 1994, MNRAS 2671035 
Waxman, E. 2003, Nature 423, 388

This preprint was prepared with the AAS $\mathrm{L}_{\mathrm{E}} \mathrm{T}$ macros v5.0. 